\title{
EL AMOR A LA GEOMETRÍA, PASIÓN SINGULAR DE UN DON JUAN NO CONVENCIONAL
}

\author{
Fernando Magallanes Latas
}

\begin{abstract}
The present article treats in depth the personality of one of the great myths in the literature of all times, in the line of the recent bibliography about Frisch's Don Juan, and aims to clarify the diverging aspects of this character in relation to the usual qualities of the traditional archetype.
\end{abstract}

El arquitecto y periodista Max Frisch, renombrado dramaturgo y narrador suizo en lengua alemana, y perspicaz observador de la realidad desde la óptica de la responsabilidad personal, es autor de la singular y divertida comedia Don Juan oder die Liebe zur Geometrie (Don Juan o el amor a la geometría) ${ }^{1}$. El curioso y desconcertante título de esta muestra centroeuropea del mito universal plantea una disyuntiva: ¿es Don Juan, con lo que esas dos palabras connotan inmediatamente de personalidad marcada por el deseo, o es un individuo cuyo interés apunta hacia lo abstracto reflejado en el estudio de la geometría? Pues bien, cuestión nada banal es la que subyace aquí: un problema de identidad personal, presentado en un texto dramático en el que cabe destacar la importancia de conceptos arropada por unos diálogos de notable agudeza en los que lo gracioso se entremezcla con un modo de sentir germánico y realista, nota esta última perceptible en la mentalidad de un personaje perfectamente escindida del papel que representa; pues si su actuación es donjuánica, teatral, ficticia, su pensamiento es diferenciado, moderno y actual. El espectador de la comedia bien puede así mostrar su perplejidad ante la afición intelectual de un Don Juan que exterioriza su desinterés por las mujeres, hasta el punto de que en el burdel de Celestina -el personaje de nuestra literatura que aprovecha Frisch en la comedia-

\footnotetext{
${ }^{1}$ Escrita en Zúrich y Nueva York en 1952 y estrenada un año después, el día 5 de mayo, en el "Schauspielhaus" de aquella ciudad suiza y en el "Schiller-Theater" de Berlín simultáneamente. Más tarde, en 1962, y si bien con escasos cambios, la obra fue reelaborada por su autor. Por otra parte, y si a la pervivencia en Zúrich de tendencias expresionistas y del teatro épico -del que, contrariamente a buena parte del resto de la obra dramática de Frisch, se separa el autor suizo con su Don Juan-, junto a la recepción de teatro actual foráneo, se une una considerable preocupación de orden existencialista en el creador del Don Juan geómetra, tenemos el basamento en el que se fraguan los dramas de zuriqués Max Frisch, escritor decididamente inclinado a la reflexión en torno a la existencia individual, interesado en planteamientos dramáticos que hagan ver al público la angustia del hombre, y uno de los autores suizos contemporáneos que, por los temas que aborda en su narrativa y en su aportación teatral, sobrepasa con mucho los estrechos límites de un localismo suizo, característico de épocas literarias pasadas. Sin renunciar a su patria -antes al contrario, apegado a su país como el que más- Frisch se sale conscientemente del reducido mundo suizo con intención de que sus compatriotas se interesen por cuestiones más generales que afectan al hombre, al ser humano, siendo buen ejemplo de ello, precisamente, su Don Juan amante de la geometría.
} 
lo que hace el Don Juan frischeano es ponerse a jugar al ajedrez. Y es que, ciertamente, esta muestra literaria suiza es bien ilustrativa de aquella afirmación del Profesor Pérez Varas: "El hecho indiscutible es que Don Juan experimenta en tierras germánicas quizás las transformaciones más trascendentales que haya sufrido tan ajetreada figura..."

El texto definitivo, que consta de cinco actos y tres "intermezzos", intercalados tras el primero, segundo y cuarto acto, presenta una vez más la legendaria figura de Don Juan, que le sirve al autor para confeccionar una trama a partir de un argumento sobradamente conocido, pero con características personales del protagonista bien distintas de las del Don Juan tradicional; un Don Juan localizado en Sevilla en época indeterminada ${ }^{3}$ y rodeado salvo alguna excepción- de conocidos personajes literarios. Nueva versión, variante o reinterpretación si se quiere, en donde se muestra un Don Juan ciertamente peculiar, amigo de la soledad, que manifiesta claramente su inclinación por la geometría, y que adquiere una repentina e inusitada fama por haber medido la fortaleza enemiga cordobesa, pero sin arriesgarse lo más mínimo gracias a su saber matemático; labor por la que es recompensado con Doña Ana, como futura esposa, a la que había conocido la noche anterior en el parque, aunque sin saber que se trataba de la hija del comendador sevillano Don Gonzalo, quien precisamente le encomendó la misión que le convierte en héroe. Pero este Don Juan, de veinte años, nada interesado en dedicar su tiempo al amor ni al juego de la intriga amorosa, como convencido que está de la mayor importancia de otras ocupaciones frente a la relación con la mujer, renunciará en el último momento al compromiso con la joven que, desesperada, decidirá acabar con su vida ahogándose en un lago.

Así pues, tras la presentación de esta paradójica personalidad de Don Juan en el primer acto, y después del "intermezzo" que le sigue con las figuras de Celestina y la prostituta Miranda -personaje inventado por Frisch-, la boda frustrada, ya en el acto segundo, será el punto de partida para la aventurera y ajetreada vida que habrá de "soportar" el protagonista en lo sucesivo.

En efecto, van a ser las consecuencias de la negativa del novio ante una boda preparada por Tenorio, su padre, y por el Comendador el motivo para la configuración de la personalidad de Don Juan o, mejor dicho, para la adjudicación del rol que habrá de desempeñar durante años: la necesidad de esconderse tras la decisión de huir le lleva al aposento de Doña Elvira, madre de Doña Ana y -como referirá el protagonista, ya en el tercer acto-, además de a otro aposento, al de Doña Inez, novia de su amigo Don Roderigo, mujeres por las que en realidad el personaje de la comedia es seducido. Pero no importa esa

\footnotetext{
${ }^{2}$ F. Pérez Varas, “Apunte sobre la fortuna de 'Don Juan' en Alemania”, Filología Moderna, № 19-20 (1965), pp. 223-245; aquí 225 .

3 "Ein theatralisches Sevilla" ("Una Sevilla teatral), apunta el autor como lugar de escenificación; y "Eine Zeit guter Kostüme" ("Una época de buenos disfraces"), indica para la representación. Por lo demás, si bien los sucesos reseñados corresponden a la Edad Media, otros elementos -como la alusión a Tirso de Molina, en el acto quinto de la comedia- sitúan la acción en una España posterior e, incluso, desde un punto de vista psicológico, el personaje da muestras de una actualidad incompatible con la época en que se supone transcurren los acontecimientos. Una cierta arbitrariedad es, pues, rasgo característico de esta comedia, no exenta de anacronismos y de relativa fidelidad histórica.
} 
realidad; el hecho es que este personaje va adquiriendo, sin embargo, fama suficiente como para engrosar la larga lista de donjuanes que presenta la literatura europea desde hace siglos.

Desde el momento de la negativa al matrimonio, este atípico Don Juan tendrá que protagonizar toda una serie de peripecias: víctima de la persecución de su frustrado suegro, será el causante, más o menos indirecto, de una sucesión de desgracias como consecuencia de verse envuelto en acciones y sucesos de los que no están ausentes ni la muerte ni el engaño o la deshonra, y que supondrán su conversión, aparentemente, en un auténtico Don Juan. Pero en un Don Juan arrastrado a ese papel -y en esto es en lo que el autor parece querer hacer particular hincapié: en el rol que nos asignan los demás ${ }^{4}$ - por la fuerza de los acontecimientos. Ello no significa, sin embargo, que nos hallemos ante un individuo inseguro; como el personaje de Tirso, Don Juan aquí está seguro de sí mismo, pero seguro no de la pasión por la conquista, que en absoluto es en este texto alemán un fin en sí, sino de su interés por la geometría, lo que le lleva a repudiar -para no ser un burlador- el tipo de vida donjuanesca al que le han conducido las propias mujeres, a las que se ha visto obligado a seducir, y los duelos inevitables en los que vence, eso sí, por pura casualidad. Es el caso, por ejemplo, de Don Gonzalo, a quien el azar convertirá en víctima accidental de la espada de Don Juan; otros se suicidarán, como la novia y anterior amante desconocida, Doña Ana, o el amigo, Don Roderigo; tampoco Tenorio, padre del "seductor", soportará la conducta de su hijo y sucumbirá como una víctima más.

Tras doce años de vida donjuanesca y harto de tal actividad, este Don Juan suizo quiere desembarazarse de su donjuanismo. A tal fin, organizará una farsa, ya en el cuarto acto, en el que aparece persuadido por Celestina para que se case con Miranda, viuda ahora del duque de Ronda. Junto a su sirviente Leporello, Don Juan espera la llegada de trece damas, que dicen haber sido seducidas por él; espera también al Obispo de Córdoba y a un invitado de excepción: el convidado de piedra -el espíritu de Don Gonzalo protagonizado aquí por la alcahueta Celestina-, con el fin de escenificar ante ese público su viaje al infierno, y así poder refugiarse, según pacta con el representante eclesiástico, en la soledad del claustro para dedicarse a su pasión: la geometría. No todo saldrá, sin embargo, como había imaginado Don Juan: aunque las mujeres crean realmente en la definitiva desaparición del seductor, éste es ahora víctima de la treta de Don Balthazar Lopez, uno de los maridos engañados que, disfrazado de obispo, había hecho el trato con Don Juan dispuesto a desbaratar los planes del libertino. Finalmente, en el último acto, al tiempo que se menciona la representación de "El Burlador de Sevilla" en los teatros sevillanos, al espectador de la obra de Frisch se le da a conocer el fin de este Don Juan a la suiza, que no será ni la condena ni la salvación de sus homónimos antecesores creados respectivamente por Tirso y

\footnotetext{
${ }^{4}$ De ahí el paralelismo de esta pieza teatral con Stiller, una de las novelas más conocidas del autor, en donde Frisch presenta el problema del hombre insatisfecho o descontento de su propia identidad. Véase al respecto, "Stillers Problem der Identität", en: Monika Wintsch-Spiess, Zum Problem der Identität im Werk Max Frischs. (Zürich: Juris-Verlag, 1965), pp. 81-93. Sobre la concomitancia de ambos textos en lo que al tratamiento de la cuestión de la identidad se refiere, véase: Manfred Jurgensen, Max Frisch. Die Dramen. (Bern und München: Francke Verlag, 1976). En concreto, dentro del capítulo V: “Appendix A: Don Juan und Stiller", pp. 46-47.
} 
Zorrilla, sino el de un hombre común, casado con Miranda, enamorada de Don Juan ya desde el primer acto, ${ }^{5}$ y con la que vivirá el resto de sus días en el castillo ducal, como padre además del hijo que ambos esperan.

El amor a la geometría es, efectivamente, pasión singular de un nuevo Don Juan nacido en el ámbito alemán. ${ }^{6}$ Por medio de un entramado de diálogos -verdadero soporte de esta obra en la que la acción ocupa un plano secundario-, Max Frisch va a utilizar en su drama, no precisamente novedoso en lo que a estructura se refiere, el mito donjuanesco; pero lo va a hacer manipulando el personaje de tal manera que su versión no resulta sino una alteración de la personalidad de Don Juan, por cuanto este protagonista germánico parece incluso ir más allá de un concepto moderno del Don Juan que atrae a las mujeres más que del Don Juan seductor y perverso. Diríase, pues, que en cuanto a localización de la acción y asunto del texto dramático, la versión frischeana se inscribe en la tradición donjuanesca; mientras que en lo referente a la personalidad de Don Juan, nos hallamos ante un individuo de mentalidad y modo de ser mucho más actual. Así, por ejemplo, el carácter teológico de la obra que dio vida al personaje mítico da paso en Frisch a una visión de la realidad más asequible al espectador de la segunda mitad del siglo XX, a través de un personaje que, si viviera hoy -dice el autor en un comentario final a su pieza dramática- se dedicaría a la física nuclear. ${ }^{7}$ En todo caso, Suiza ${ }^{8}$ contribuye de esta manera y a través de uno de sus escritores más internacionales a la pervivencia, ampliación y actualización de la literatura donjuánica ${ }^{9}$; a la expansión de uno de los temas más afianzados en distintas literaturas y épocas históricas, y a la difusión de una personalidad con genuinas señas identificativas ${ }^{10}$,

\footnotetext{
${ }^{5}$ Cuando éste jugaba al ajedrez, por ser "el primer hombre que tuvo el valor de hacer lo que a él se le antojó incluso en el burdel", dice Miranda. Max Frisch, Gesammelte Werke. Dritter Band. (Frankfurt am Main: Suhrkamp Verlag, 1986), p. 102.

${ }^{6}$ Entre otras, destacadas versiones del tema de Don Juan en la literatura alemana son las de Christian Dietrich Grabbe, escritor de las primeras décadas del siglo pasado y autor de Don Juan und Faust (Don Juan y Fausto), tragedia en cuatro actos; la de Nikolaus Lenau, que escribió, en 1844, el poema dramático Don Juan; la del Premio Nobel de 1910, Paul Heyse, autor de Don Juans Ende (El fin de Don Juan); o el drama del expresionista Carl Sternheim, de 1910, Don Juan.

${ }^{7}$ M. Frisch, op. cit., p. 173. Sobre esta cuestión, véase la obra del Profesor Carlos Buján López: La figura del físico atómico en el teatro alemán contemporáneo. Universidad de Salamanca, 1979.

${ }^{8}$ La producción teatral de ese país, fundamentalmente en lenguas francesa y alemana, y en el caso de esta última y salvo rara excepción en dialecto suizo- en idioma alemán estándar, encuentra en Zúrich, precisamente ciudad natal de Max Frisch, su lugar más relevante para la proyección y difusión de la creación dramática. Allí se estrenaron obras de teatro épico de Brecht -línea en la que hay que inscribir, en parte, al autor del Don Juan amante de la geometría-, del expresionista Georg Kaiser y de otros dramaturgos alemanes durante el dominio nacionalsocialista; y allí, también, hay que situar el punto de arranque de la actividad teatral en lengua alemana tras el año de la catástrofe, el año 1945.

${ }^{9}$ Independientemente de las no pocas opiniones negativas (tai vez por desconocimiento de la realidad alemana o por temor pueril a que un autor extranjero pueda dañar la imagen de nuestra legendaria figura donjuanesca) vertidas en España en torno a esta obra de Frisch con ocasión de las representaciones que de ella se realizaron en Madrid (1968/1972) y en Valladolid (1968). Véase al respecto: Antonio Fernández de Gorostiza Foronda, Max Frisch: su recepción en España. Valoración crítica, (Madrid: Editorial de la Universidad Complutense, 1990), en donde se ofrece una recopilación exhaustiva de la crítica española sobre el Don Juan de Frisch.

${ }^{10}$ Cfr.: Claudio Guillén, Entre lo uno y lo diverso. Introducción a la literatura comparada. (Barcelona: Editorial Crítica, 1985), pp. 110-111.
} 
que en la variante frischeana, lejos de corresponder a una "figura estática", propia de otras versiones literarias del mito, ofrece una trayectoria evolutiva ${ }^{11}$ que se inicia con la adjudicación de un papel, cuya principal implicación es el progresivo distanciamiento del protagonista respecto de su pasión: la geometría; y culmina en el abandono voluntario de ese rol y en el consiguiente retorno de Don Juan a su ocupación matemática, tras un lapso de tiempo de doce años durante el cual ha tenido que desarrollar una actividad no acorde con su propia voluntad.

Se trata por tanto, en este caso, de una personalidad desviada hacia una conducta no deseada, cuestión de no poco interés para un intelectual, como Frisch, inquieto y desasosegado por el lado falso de las personas, y que resalta especialmente en una comedia en la que lo efectista y la teatralización del mito contribuye de manera muy considerable a sus propósitos. No en vano este novelista escribe también teatro convencido de las posibilidades que ofrece la escena de mayor aproximación al público, a ese destinatario al que quiere tener presente a la hora de escribir.

Rol, máscara o identidad son, por consiguiente, términos clave en su obra literaria, en la que esta nueva versión de Don Juan no es ninguna excepción: tanto en su sentido literal -las máscaras de la fiesta de disfraces inicial o el personaje Don Balthazar Lopez que, en el acto cuarto, aparece camuflado haciéndose pasar por obispo-, como en el sentido de rol culpable del enmascaramiento de la identidad personal, tal y como sucede a un Don Juan que, violentada por los demás su personalidad, poco tiene que ver con la ostentación escandalosa y la consciente amoralidad del Don Juan tradicional. ${ }^{12}$ Por eso Frisch contribuye a la generalización de un mito que, si bien puede haber nacido en el ámbito meridional, es ya patrimonio universal. Pero el personaje mítico, al universalizarse, adquiere cualidades nuevas, distintas, de manera que el prototipo originario pierde ya en su versión suiza no pocas de sus cualidades peculiares, desde el momento en que, si bien se trata de un individuo que enamora -o del que se enamoran- con facilidad las mujeres, su meta en la vida, su vocación, no es enamorar y abandonar una y otra vez, porque su auténtico amor es la geometría, no la apetencia desaforada o el goce momentáneo. No es de extrañar, por tanto, que Manfred Schmeling ${ }^{13}$ hable -utilizando terminología del teórico de la Recepción Literaria, Hans Robert Jauss- de la ruptura del horizonte de expectativas del público cuando se refiere al Don Juan de Frisch; porque, en efecto, se trata aquí de un Don Juan a la fuerza, de un Don Juan que ama, no a las mujeres, sino la geometría, de un individuo que representa no un modo de ser, sino un papel impuesto por los demás, impuesto por las mujeres en este caso: Don Juan es realmente "la víctima de un papel"14 que, por la fuerza

\footnotetext{
${ }^{11}$ Cfr.: Manfred Durzak, Dürrenmatt, Frisch, Weiss. Deutsches Drama der Gegenwart zwischen Kritik und Utopie. (Stuttgart: Philipp Reclam jun., 1972), pp. 196-207.

${ }^{12}$ Véase: Gregorio Marañón, Don Juan. Ensayos sobre el origen de su leyenda. (Madrid: Espasa-Calpe. Colección Austral. Novena edición, 1960), p. 80.

${ }^{13}$ Cfr.: Manfred Schmeling, Teoría y praxis de la literatura comparada. (Barcelona: Editorial Alfa. Estudios Alemanes, 1984), pp. 91 y ss.

14 "Er ist das Opfer einer Rolle". Wolfram Budecke und Helmut Fuhrmann, Das deutschsprachige Drama seit 1945. Schweiz. Bundesrepublik. Österreich. DDR. Kommentar zu einer Epoche. (München: Winkler Verlag,
} 
del equívoco y del malentendido, tiene que sobrellevar; la posibilidad de ejercer la libertad personal se halla por tanto muy limitada en este personaje que se ve continuamente obligado a desempeñar el rol que le ha correspondido.

En consecuencia, no sería exagerado mencionar el texto suizo como ejemplo genuino de desviación donjuanesca, porque lo que en realidad hace el dramaturgo en este caso es retomar la trayectoria literaria del personaje para darle la vuelta al individuo seductor y convertirlo en lo contrario, en un Don Juan seducido por el entorno, en un hombre que no persigue lo que realmente está protagonizando, sino que son los acontecimientos en los que participa los que le vienen rodados, inesperadamente impuestos. Obra desconcertante, pues, de la que con razón se ha dicho ${ }^{15}$ que es sorprendente y humorístico a la vez el hecho de que Don Juan termine por cansarse de ser víctima de la seducción femenina, hasta el punto de ansiar el mundo de la pura abstracción matemática y de la claridad espiritual. Y, de ahí, el que en ocasiones también la crítica se haya referido al autor suizo como a un escritor que ha creado un no-Don Juan; un anti-Don Juan en un texto dramático que, en cierto modo, se situaría en las antípodas de la literatura donjuánica tradicional; un no-Don Juan porque anímicamente no se siente Don Juan; si actúa como tal es porque no le queda más remedio, no porque el personaje creado por Frisch experimente una tendencia instintiva o irresistible hacia el comportamiento donjuanesco.

Ésta es la diferencia esencial entre el Don Juan suizo y el arquetipo tradicional; diferencia que permite pensar en el individuo de la comedia de Frisch como en un no-Don Juan. El no-donjuanismo de la comedia suiza es, pues, consecuencia del tipo de personalidad que presenta el protagonista, no de su trayectoria o de su destino; y, en este sentido, es comprensible lo dicho por Manfred Schmeling ${ }^{16}$ : "Quien (...) razone que el Don Juan de Frisch no es Don Juan porque se ha casado y es padre, no ha aprendido nada de la 'parábola' de Frisch: sigue adherido a una imagen de Don Juan y no quiere liberarlo de su papel."17

1981), p. 30. Y, en efecto, se dan

en el personaje de Frisch con toda precisión las caracterís-

ticas más genuinas del rol social a tenor de las precisiones

que, sobre el concepto de papel, apuntó en su día la Sociolo-

gía alemana: En primer lugar, el papel implica prescripciones de conducta que son independientes del individuo; en segundo lugar, su contenido no lo determina o modifica el individuo, sino la sociedad; y, en tercer lugar, el individuo no puede soslayar las expectativas de comportamiento que se exigen de cada papel, a menos de resultar perjudicado. Ralf Dahrendorf,

Homo sociologicus. Ein Versuch zur Geschichte, Bedeutung und

Kritik der Kategorie der sozialen Rolle. Studienbücher zur

Sozialwissenschaft. Band 20. (Opladen: Westdeutscher Verlag,

1977), p. 35.

${ }^{15}$ Cfr.: Helmut Prang, Geschichte des Lustspiels. Von der Antike bis zur Gegenwart. (Stuttgart: Alfred Kröner Verlag, 1968), p. 351.

${ }^{16}$ M. Schmeling, op. cit., p. 96.

${ }^{17}$ En todo caso, y pese a las aclaraciones del propio autor en su "Nachträgliches zu 'Don Juan" ("Apéndice sobre 
Así pues, el Don Juan de Frisch actúa como un Don Juan entendido en su sentido vulgar o tradicional, pero si lo hace no es sino impelido por el entorno, no porque esa actuación se la dicte su instinto primitivo, rudimentario, casi animalesco o indiferenciado ${ }^{18}$ como sucede en el Don Juan ocupado en seducir, insaciable y despectivo con la mujer, protagonista de otras obras impulsoras del mito. Este Don Juan germánico protagonizará, efectivamente, peripecias propias y típicas de la literatura donjuanesca; pero lo hará involuntariamente, forzado por unas circunstancias que, de forma concatenada, le llevan a cometer actos propios de lo que se concibe tradicionalmente por modo de ser donjuánico. Por eso, el motivo literario de la seducción, que adquiere su especificidad más genuina en la literatura donjuanesca, pierde consistencia en esta obra suiza y se convierte en elemento de una trama argumental que solapa los verdaderos propósitos de un autor preocupado por las interferencias del rol social en la identidad personal.

Como en otras versiones del mito, ofrecidas en la literatura europea, este Don Juan suizo pierde aquella voluptuosidad y cinismo del personaje de Tirso y pasa a ser un individuo desinteresado por la conquista, carente de intencionalidad perversa, encarnación, no de lo demoníaco o sacrílego, sino de la pura intelectualidad, por mucho que, aparentemente, puedan pensar de él lo contrario la Iglesia, las damas seducidas o los maridos engañados por el geómetra. Y esa intelectualidad es la nota definitoria más atinada, expresada por el propio autor: Don Juan es un intelectual; un ser reflexivo que bien puede admirar al enemigo árabe por su superioridad de conocimientos matemáticos; un hombre que medita y conversa sobre asuntos como la cuarta dimensión; un apasionado del estudio, manifestado a través 'de su amor por la geometría; por la matemática como sinónimo de autocontrol, es decir, precisamente lo opuesto, el contrapunto de un Don Juan convencional, libertino, anárquico, primitivo...

Cabe, así, pensar en la figura creada por Frisch como en un Don Juan con personalidad germana, tal vez más que -como reiteradamente se ha dicho- en una desmitificación del personaje mítico; y no sólo por la peculiar configuración de este nuevo Don Juan, en el que quizá se aúnen cualidades de distintos personajes del mismo nombre creados por la ficción literaria, sino que además las reflexiones de su autor en el comentario a modo de apéndice final bien pueden apuntar a un engarce del Don Juan latino en la mentalidad alemana.

Sería, en tal caso, una obra pensada y escrita para un público germánico en donde un personaje Don Juan exterioriza la rebeldía inherente a esta figura tradicional, pero más que ante las normas de la convivencia social, de la moralidad o de la Religión, se rebela contra lo que los demás piensan de él, contra el papel que se le ha asignado y del que desea liberarse como vía para profundizar en su propia identidad. El autoconocimiento para poder ser uno mismo en la vida es, en definitiva, lo que le preocupa a Don Juan; de hecho, su enamorada de siempre, Miranda, ya como duquesa de ronda, le dice sin rodeos que sólo se

Don Juan"), de 1953, las posibles interpretaciones de esta nueva figura donjuánica deberían quedar, si cabe, más abiertas, si nos atenemos a la constante intención literaria de su autor de inhibirse a la hora de ofrecer soluciones a los problemas planteados.

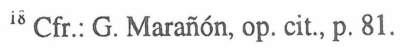


ha amado a sí mismo sin conseguir, en cambio, encontrarse, y que por eso en realidad él Don Juan- odia a las mujeres; el único camino hacia la geometría es entregarse a una sola mujer. ${ }^{19}$ Y, efectivamente, Don Juan es consciente de esa falta de autoconocimiento: "no sé si soy distinto a otros hombres" ${ }^{\prime 20}$, dice en determinado momento de la obra.

Así pues, y a través del personaje mítico, el escritor suizo incide en la relatividad de toda individualidad desde el momento en que tiene lugar un proceso de socialización y se produce la integración en una colectividad; el impedimento o la traba que supone tal incorporación para la singularidad del individuo lo lleva Frisch a extremos grotescos ejemplificando la cuestión en un personaje que ve disminuida su especificidad individual como consecuencia del ambiente y de las circunstancias que le rodean.

En definitiva, tanto si se admite una genuina personalidad donjuánica en este personaje, como si se le califica de no-Don Juan, lo cierto es que se trata de un individuo que no quiere ser Don Juan; lo que quiere es que le dejen vivir tranquilo dedicado al estudio de la geometría; ${ }^{21}$ Don Juan es así un hombre que adopta una actitud, un papel o un comportamiento que repele su propia subjetividad, y, al obrar de este modo, el problema que se plantea es de pérdida de identidad; y eso es, al fin y al cabo, lo importante, lo que ha de tenerse en cuenta.

Consecuentemente, el texto presenta dos realidades: una encarnada en Don Juan, así llamado un individuo que se automargina ${ }^{22}$, que si piensa o siente como el arquetipo español tradicional lo es sólo de manera forzada; es, diríase, la representación de una mentalidad y forma de ser extraña a la personalidad donjuánica meridional; mientras que la otra realidad presente en la comedia es la que muestra el resto de los personajes de la obra, extraídos en su mayoría de literatura donjuánica previa, que revelan claramente el interés hacia el mito por parte del dramaturgo suizo. Y ambas realidades, tradición literaria donjuanesca y sensibilidad impropia del modo de ser donjuánico, chocan permanentemente a lo largo de los cinco actos dando como resultado unas reacciones de este Don Juan perfectamente comprensibles; de ahí que pudiera no ser tanta, como en ocasiones se ha dicho, la intención del autor de desmitificar, de ironizar, de presentar una farsa o incluso, podría decirse, de ridiculizar a un Don Juan tradicional. Cuestión naturalmente distinta es que el público o lector no familiarizado con la sensibilidad germana perciba los comentarios como con una dosis mayor de ironía de la que en realidad tal vez pretendió hacer uso el autor, o se imagine a este singular Don Juan como un individuo ingenuo, sin

\footnotetext{
${ }^{19}$ M. Frisch, op. cit., p. 145.

${ }^{20}$ M. Frisch, op. cit., p. 155.

${ }^{21}$ Por eso, en expresión de Maurice Molho, que considera la obra como "la más profunda parodia-síntesis del mito", "La tragedia de Don Juan es verse inserto en un mundo no geométrico, sustraído a la necesidad". Maurice Molho, Mito-

logías. Don Juan. Segismundo. (Madrid: Siglo XXI, 1993), pp. 192 y 196.

${ }^{22} \mathrm{O}$, como dice Adelheid Weise en su trabajo doctoral Untersuchungen zur Thematik und Struktur der Dramen von Max Frisch. (Göppingen: Verlag Alfred Kümmerle, 1975), p. 83, "Don Juan está fuera de la realidad".
} 
que ello quiera significar una merma ni reste un ápice a lo que de burla y diversión tiene la obra.

En conclusión, es un problema de limitación del desarrollo personal y de la exteriorización de la propia subjetividad el que plantea esta pieza humorística: la dificultad de ejercer la libertad, de llevar a cabo lo que uno realmente desea, reside en la enorme fuerza constrictora que ejerce el papel que el individuo ha de representar, en buena medida involuntariamente; Don Juan en Frisch actúa, por tanto, tal y como se espera que lo haga, evidenciando de manera más fingida de lo usual eso que las ciencias sociales denominan fachada o "parte de la actuación del individuo que funciona regularmente de un modo general y prefijado, a fin de definir la situación con respecto a aquellos que observan dicha actuación" ${ }^{23}$ Es como si el dramaturgo suizo quisiera adelantarse en dos décadas a lo que fue ocupación habitual de escritores en lengua alemana: el interés, incluso preocupación, en torno a problemas relativos a la crisis de identidad, la autoindagación y el afán por descubrir y exteriorizar matices de la personalidad desde una perspectiva puramente intimista. El mérito fundamental de Frisch consiste, pues, en haber tenido la habilidad suficiente para tratar un tema más que serio en una obra jocosa, incluso aparentemente superficial; en una obra especialmente relevante por la destreza de que hace gala el autor en su replanteamiento del tema de Don Juan. También, y aunque sin detenerse en profundas disquisiciones, Frisch aborda temas de envergadura y trascendencia considerables, como cuando Don Juan manifiesta no entender la Creación ${ }^{24}$ y se pregunta acerca de la división de sexos del género humano: ¿por qué esa distinción entre hombre y mujer? ¿Qué sentido tiene para la existencia la realidad de dos sexos diferentes? Por lo demás, lo que hace Frisch es recrear un tema archiconocido, teatralizar -recuérdese el montaje teatral preparado por el propio Don Juan para desprenderse de su papel de libertino- una historia en la que se intercalan elementos novedosos, originales, realistas, serios e importantes en una estructura basada en la fuerza del diálogo chistoso, burlón y, sobre todo, entretenido.

${ }^{23}$ En consecuencia, "la fachada (...) es la dotación expresiva de tipo corriente empleada intencional o inconscientemente por el individuo durante su actuación". Ervin Goffman, La presentación de la persona en la vida cotidiana. (Buenos Aires: Amorrortu editores, 1981), pp. 33-34.

${ }^{24}$ M. Frisch, op. cit., p. 146. 
